\title{
Subtipos del síndrome de intestino irritable en niños
}

\section{Subtypes of irritable bowel syndrome in children}

\author{
Carlos Alberto Velasco-Benítez ${ }^{1}$ (D), Jairo Enrique Moreno-Gómez ${ }^{2}$ (D), Carmen Rossy Ramírez-Hernández ${ }^{3}$ (D)
}

1. Universidad del Valle. Cali, Colombia. Correo: carlos.velasco@correounivalle.edu.co - https://orcid.org/0000-0002-4062-5326

2. Clínica Pediátrica Colsanitas. Bogotá, Colombia. Correo: jemoreno@colsanitas.com - https://orcid.org/0000-0002-1927-7881

3. Hospital María Inmaculada. Florencia, Colombia. Correo: carmessy@gmail.com - https://orcid.org/0000-0003-1180-9086

Tipología: Artículo de investigación científica y tecnológica

Para citar este artículo: Velasco-Benítez CA, Moreno-Gómez JE, Ramírez-Hernández CR. Subtipos del síndrome de intestino irritable en niños. Duazary. 2020 enero; 17(1): 62 73. Doi: http://dx.doi.org/10.21676/2389783X.3223

Recibido en enero 15 de 2018

Aceptado en junio 28 de 2019

Publicado en línea en noviembre 19 de 2019

\section{RESUMEN}

\begin{abstract}
Palabras clave: enfermeda des del sistema digestivo; síndrome del colon irritable; subtipo; niño.
\end{abstract}

La prevalencia del síndrome de intestino irritable (SII) pediátrico es $1,2 \%-5,4 \%$ y los subtipos en niños latinoamericanos no están especificados. El objetivo de esta investigación es caracterizar los subtipos del SIl y sus posibles asociaciones mediante un estudio de prevalencia en niños entre los 8 y 18 años de edad con SII según los Criterios de Roma III. Se tomaron variables sociodemográficas, familiares y clínicas. Los subtipos de SII se consideraron por la consistencia de las heces en SII con estreñimiento (SII-e); diarrea (SIId); mixto (SII-m) y sin subtipo (SII-ss). La estadística incluyó análisis uni y bivariados. Los datos fueron analizados usando t-Student a dos colas, chi cuadrado, prueba exacta de Fisher y razón de prevalencia (RP) con IC 95\%, siendo $p<0,05$ significativa. Fueron incluidos 196 niños (53,6\% femeninos; mediana de 11 años) que presentaron SII-ss en $64,8 \%$, SII-e en $19,9 \%$, SII-m en $8,7 \%$ y SII-d en $6,6 \%$. Hubo diferencias significativas en las características de las heces $(p=0,01)$, en la incontinencia fecal $(p=0,02)$ y en los fecalomas $(p=0,00)$, así como posibles asociaciones entre el SII- $d$, la ciudad $(p=0,00)$ y malnutrición $(p=0,02)$. En conclusión, luego del SII-ss, un cuarto de los niños estudiados presentó SII-e, seguido del SII-m y del SII-d, este último con mayor oportunidad en malnutridos.

\section{ABSTRACT}

Keywords: Digestive system diseases; Irritable bowel syndrome; Subtype; Child.
The prevalence of Irritable Bowel Syndrome (IBS) in children is $1.2 \%-5.4 \%$, and the subtypes in Latin American children has not been specified. The objective of this study is to characterize the different subtypes of IBS in children and their possible associations. A prevalence study was carried out in Colombian children between 8-18 years of age with IBS according to the Rome III Criteria. Sociodemographic, family and clinical variables were taken. The subtypes of IBS were considered depending on the consistency of stool in IBS with constipation (IBS-c); with diarrhea (IBS-d); mixed (IBS-m) and without subtype (IBS-u). The statistics included uni and bivariate analyzes. The data were analyzed using two-tailed student $t$-test, chi $^{2}$ test, Fisher's exact test and $P R$ with $95 \% \mathrm{Cl}$, being $\mathrm{p}<0.05$ significant. 196 children were included (53.6\% female, median 11 years old); presenting IBS-u in 64.8\%, IBS-c in $19.9 \%$, IBS-m in $8.7 \%$ and IBS- $d$ in $6.6 \%$. There were only significant differences in stool characteristics $(p=0.01)$, in the fecal incontinence $(p=0.02)$ and the fecalomas $(p=0.00)$. There were possible associations between children with IBS- $d$, city $(p=0.00)$ and malnutrition $(p=0.02)$. In conclusion, after the IBS- $u, 1 / 4$ of the schoolchildren and adolescents studied presented IBS-c, followed by IBS-m and IBS-d; presenting IBS-d with greater opportunity in malnourished children. 


\section{INTRODUCCIÓN}

Para diagnosticar los desórdenes gastrointestinales funcionales (DGF) en escolares y adolescentes se ha propuesto la guía de síntomas de los Criterios de Roma $^{1}$. La prevalencia de los DGF en escolares y adolescentes colombianos es del $23,7 \%$, siendo en orden de presencia los más frecuentes el estreñimiento funcional en un $11,6 \%$, el síndrome de intestino irritable en un $5,1 \%$, el dolor abdominal en un $2,5 \%$ y la migraña abdominal en el $1,7 \%$, entre otros. Estos desórdenes se presentan más en los escolares entre los 8 y 12 años de edad, del género femenino, de colegio privado $y$ con padres separados/divorciados $(p<0,05)^{2}$.

Según los Criterios de Roma IV ${ }^{1}$, para presentar síndrome de intestino irritable (SII) se debe incluir todo lo siguiente, al menos dos meses antes del diagnóstico: 1) dolor abdominal al menos por cuatro días por un mes asociado con uno o más de lo siguiente: a) la defecación, b) cambios en la frecuencia de las heces, y c) cambio en la forma (apariencia) de las heces; 2) en los niños con estreñimiento funcional, el dolor no se resuelve con la resolución del estreñimiento (niños en quienes el dolor se resuelve tienen estreñimiento funcional y no SII), y 3) después de una apropiada evaluación, los síntomas no pueden ser explicados por otra condición médica.

La prevalencia del SII en niños ha sido reportada entre un $1,2 \%$ y $5,4 \%^{3-5}$. En niños colombianos, no hay predominio del género femenino para presentar SII; sin embargo, su prevalencia es mayor en los escolares entre los 8 y 12 años de edad ${ }^{6}$.

En adultos la clasificación de los subtipos del SII incluye: SII con estreñimiento (SII-e), SII con diarrea (SII-d), SII mixto (SII-m) y SII sin subtipo (SII-ss) ${ }^{7-12}$. No obstante, por medio de los Criterios de Roma III, tal clasificación no ha sido especificada para niños $^{13}$. La identificación de los subtipos del SII en niños comienza a ser importante tanto en la investigación como en la clínica debido a que el manejo farmacológico del SII empieza a ser más específico y las nuevas terapias están siendo desarrolladas dependiendo del subtipo específico de SII.
El objetivo del presente estudio es caracterizar los diferentes subtipos del SII en niños colombianos y sus posibles asociaciones.

\section{MATERIALES Y MÉTODOS}

\section{Tipo de estudio}

Estudio descriptivo observacional, donde se tuvieron en cuenta variables sociodemográficas (edad, sexo), familiares (hijo único, padres separados/divorciados, DGF intrafamiliares) y clínicas (peso, talla, malnutrición, talla alterada). Se clasificaron en escolares entre los 8 y 12 años de edad y adolescentes entre los 13 y 18 años de edad. Se definió malnutrición y talla alterada para la edad según el AnthroPlus de la Organización Mundial de la Salud.

\section{Población y muestra}

Estudio no experimental descriptivo observacional de corte transversal tipo prevalencia realizado en 12 colegios (8 colegios públicos y 4 colegios privados) de tercero a once grado de 10 ciudades colombianas (Bogotá, Distrito Capital; Cali, Valle; Cartagena, Bolívar; Cúcuta, Santander; Florencia, Caquetá; Pereira, Risaralda; Santander de Quilichao, Cauca; Soledad, Atlántico; San Andrés de Sotavento, Córdoba y La Unión, Nariño), de cuatro regiones colombianas (andina, atlántica, pacífica y amazónica). Los datos fueron tomados de la base de datos del Grupo FINDERS (Functional International Digestive Epidemiological Research Survey) con diagnóstico de SII.

\section{Procedimientos e instrumentos}

Los niños respondieron el Cuestionario para Síntomas Gastrointestinales Pediátricos Roma III para Escolares y Adolescentes (QPGS-III) para identificar DGF, el cual ha sido validado y probado en español ${ }^{14}$. Los niños entre 8 y 10 años lo hicieron de manera guiada con uno de los investigadores principales, y los niños entre los 11 y 18 años por autorreporte.

EI SIl fue diagnosticado usando los Criterios de Roma III para DGF pediátricos, que debe incluir todo lo siguiente, una vez por semana durante al menos los dos últimos meses antes del diagnóstico: 
malestar abdominal (sensación incómoda no descrita como dolor) o dolor asociado con dos o más de lo siguiente, al menos en el $25 \%$ del tiempo: mejora con la defecación, inicio asociado con cambios en la frecuencia o forma (apariencia) de las heces, sin evidencia de algún proceso inflamatorio, anatómico, metabólico o neoplásico que explique dichos síntomas.

Arbitrariamente, los subtipos de SII fueron considerados dependiendo de las respuestas dadas en el QPGS-III para identificar DGF relacionadas con la consistencia de las heces durante los últimos dos meses, así: SII-e cuando las heces eran muy duras o duras; SII-d cuando las heces eran blandas, muy blandas o líquidas; SII-m cuando las heces no siempre eran iguales, y SII-ss cuando las heces no eran ni tan duras ni tan blandas.

\section{Análisis estadístico}

La base de datos fue construida con el programa Excel. Para evaluar la posibilidad de errores en la digitación se revisó un $10 \%$ de la base de datos y se contrastó con las encuestas. El análisis estadístico de los datos se realizó con el programa Stata 15.0 software (StataCorp, College Station, Texas), y para describir la distribución de las variables se llevó a cabo un análisis exploratorio de todas las variables. En el caso de las variables continuas se obtuvieron las gráficas (histogramas, cajas y bigotes, gráficos de simetría) que permitieron describir su distribución, así como las medidas de tendencia central y de dispersión. También se realizó un análisis de normalidad mediante los valores y de sesgo y curtosis con su respectiva prueba, y además se observó la transformación que mejor se acomodó a los datos para su normalización. En el caso de variables categóricas se obtuvieron frecuencias y proporciones. La prevalencia se determinó mediante la estimación de la proporción por ocurrencia y el cálculo de intervalos de confianza al 95,0\%.

Para establecer las posibles asociaciones y no factores de riesgo por no tratarse de un estudio de casos y controles, se realizó un análisis univariado entre la variable exposición y la variable efecto. En el caso de las variables categóricas se utilizó chi cuadrado, además de la asociación de la variable exposición y otras covariables, y entre la variable efecto y las demás covariables para evaluar confusión mediante la construcción de tablas de $2 \times 2$ y la estimación de las razones de prevalencia (RP) con sus respectivos intervalos de confianza $(95,0 \%)$. Para valorar la significancia estadística se utilizó la prueba exacta de Fisher $(p<0,05$, a dos colas).

\section{Declaración sobre aspectos éticos}

Según la Resolución 8430 de octubre 4 de 1993 del Ministerio de Salud Colombiano, artículo 11, este trabajo se clasifica como sin riesgo. Este estudio fue aprobado por el comité de ética de la Universidad del Valle de Cali, Colombia, según Acta de Aprobación N. ${ }^{\circ}$ 04-013 de 26/02/2013. Además, siguiendo las exigencias estipuladas en la Declaración de Helsinki, se garantizaron los derechos y el bienestar de cada participante. También se expuso de forma explícita en un documento de consentimiento informado para los padres $y / o$ tutores $y$ en un documento de asentimiento informado para los niños mayores de ocho años que los datos obtenidos iban a ser utilizados en una investigación, asegurando la confidencialidad, el manejo profesional de la información y los resultados derivados del estudio.

\section{RESULTADOS}

De los 4.394 cuestionarios de la base de datos del Grupo FINDERS, reunieron los Criterios de Roma III para SII 196 niños (53,6\% femeninos; mediana de 11 años, rango intercuartil 3; 68,4\% escolares; $73,5 \%$ de colegio público; $20,4 \%$ de Soledad, Atlántico; 39,2\% de la región andina; $48,4 \%$ con padres separados/divorciados; $9,9 \%$ con DGF intrafamiliares; $8,9 \%$ hijos únicos; $32,8 \%$ malnutridos según índice de masa corporal, y $7,8 \%$ con talla alterada para la edad). El SII-ss $(64,8 \%)$ fue el subtipo más frecuente, seguido del SII-e (19,9\%), el SII-m (8,7\%) y el SII-d (6,6\%) (Tabla 1$)$. 
Tabla 1. Características generales de niños con síndrome de intestino irritable ( $N=196)$.

\begin{tabular}{|c|c|c|c|}
\hline \multicolumn{2}{|c|}{ Características } & $\mathbf{n}$ & $\%$ \\
\hline \multirow[t]{2}{*}{ Grupos de edad } & Escolares (8-12 años) & 134 & 68,4 \\
\hline & Adolescentes (13-18 años) & 62 & 31,6 \\
\hline \multirow[t]{2}{*}{ Sexo } & Femenino & 105 & 53,6 \\
\hline & Masculino & 91 & 46,4 \\
\hline \multirow[t]{2}{*}{ Colegio } & Público & 144 & 73,5 \\
\hline & Privado & 52 & 26,5 \\
\hline \multicolumn{4}{|c|}{ Región/ciudad } \\
\hline \multirow[t]{4}{*}{ Andina } & Pereira, Risaralda & 33 & 16,8 \\
\hline & La Unión, Nariño & 22 & 11,2 \\
\hline & Cúcuta, Santander & 18 & 9,2 \\
\hline & Bogotá, Distrito Capital & 4 & 2,0 \\
\hline \multirow[t]{3}{*}{ Atlántica } & Soledad, Atlántico & 40 & 20,4 \\
\hline & Cartagena, Bolívar & 16 & 8,2 \\
\hline & San Andrés de Sotavento, Córdoba & 8 & 4,1 \\
\hline \multirow[t]{2}{*}{ Pacífica } & Cali, Valle & 31 & 15,8 \\
\hline & Santander de Quilichao, Cauca & 9 & 4,6 \\
\hline Amazónica & Florencia, Caquetá & 15 & 7,7 \\
\hline \multirow[t]{3}{*}{ Antecedentes familiares } & Padres separados/divorciados & 91 & 48,4 \\
\hline & DGF intrafamiliares & 19 & 9,9 \\
\hline & Hijo único & 17 & 8,9 \\
\hline \multicolumn{4}{|c|}{ Estado nutricional } \\
\hline \multirow[t]{2}{*}{ Según IMC (n=192) } & Eutrófico & 129 & 67,2 \\
\hline & Malnutrición & 63 & 32,8 \\
\hline \multirow[t]{2}{*}{ Según TE (n=192) } & Eutrófico & 177 & 92,2 \\
\hline & Talla alterada & 15 & 7,8 \\
\hline \multirow[t]{4}{*}{ Subtipos del SII } & SII sin subtipo & 127 & 64,8 \\
\hline & SIl con estreñimiento & 39 & 19,9 \\
\hline & SII con estreñimiento y diarrea & 17 & 8,7 \\
\hline & SIl con diarrea & 13 & 6,6 \\
\hline
\end{tabular}

SII=síndrome de intestino irritable; DGF=desórdenes gastrointestinales funcionales; TE=talla para la edad.

Las características del dolor abdominal, de las heces y de los síntomas digestivos y extradigestivos de los niños con los diferentes subtipos del SIl se muestran en las siguientes 3 tablas (Tabla 2, Tabla 3 y Tabla 4). Solo se presentaron diferencias significativas en las características de las heces ( 3 a 6 veces por semana, $p=0,01)$, y en la presencia de incontinencia fecal $(p=0,02)$ y de fecalomas $(p=0,00)$. De los 196 niños con SII, 90 faltaron al colegio o dejaron de hacer sus actividades diarias, $y$ 106 siguieron su rutina escolar $(p=0,56)$. 
Tabla 2. Características del dolor abdominal de acuerdo a los subtipos de síndrome de intestino irritable en niños ( $\mathrm{N}=196)$.

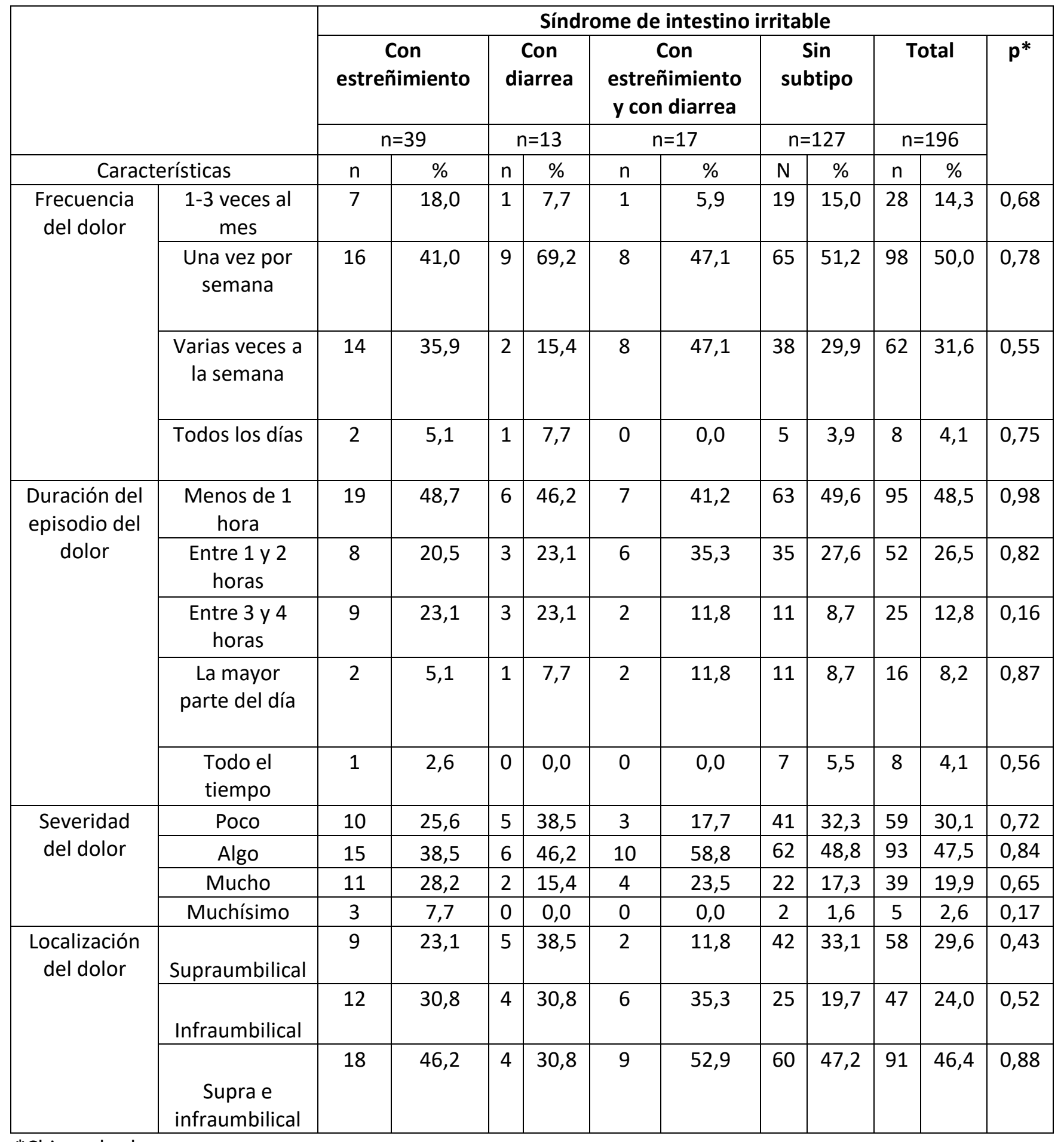

\footnotetext{
*Chi cuadrado.
} 
Subtipos del síndrome de intestino irritable en niños

Tabla 3. Características de las heces de acuerdo a los subtipos de síndrome de intestino irritable en niños ( $N=196)$.

\begin{tabular}{|c|c|c|c|c|c|c|c|c|c|c|c|c|}
\hline \multirow{4}{*}{\multicolumn{2}{|c|}{ Características }} & \multicolumn{11}{|c|}{ Síndrome de intestino irritable } \\
\hline & & \multirow{2}{*}{\multicolumn{2}{|c|}{$\begin{array}{c}\text { Con } \\
\text { estreñimiento } \\
n=39 \\
\end{array}$}} & \multirow{2}{*}{\multicolumn{2}{|c|}{$\begin{array}{c}\begin{array}{c}\text { Con } \\
\text { diarrea }\end{array} \\
n=13 \\
\end{array}$}} & \multirow{2}{*}{\multicolumn{2}{|c|}{$\begin{array}{c}\text { Con } \\
\text { estreñimiento } \\
\text { y con diarrea } \\
n=17 \\
\end{array}$}} & \multirow{2}{*}{\multicolumn{2}{|c|}{$\begin{array}{c}\text { Sin subtipo } \\
n=127\end{array}$}} & \multirow{2}{*}{\multicolumn{2}{|c|}{$\begin{array}{l}\text { Total } \\
n=196 \\
\end{array}$}} & \multirow[t]{3}{*}{$\mathbf{p}^{*}$} \\
\hline & & & & & & & & & & & & \\
\hline & & $\mathrm{n}$ & $\%$ & $\mathrm{n}$ & $\%$ & $\mathrm{n}$ & $\%$ & $\mathrm{n}$ & $\%$ & $\mathrm{n}$ & $\%$ & \\
\hline \multirow[t]{5}{*}{$\begin{array}{c}\text { Frecuencia } \\
\text { de las } \\
\text { deposiciones }\end{array}$} & $\begin{array}{c}2 \text { o menos } \\
\text { veces a la } \\
\text { semana }\end{array}$ & 23 & 59,0 & 1 & 7,7 & 7 & 41,2 & 68 & 53,5 & 99 & 50,5 & 0,165 \\
\hline & $\begin{array}{c}3 \text { a } 6 \\
\text { veces por } \\
\text { semana }\end{array}$ & 4 & 10,3 & 6 & 46,2 & 0 & 0,0 & 15 & 11,8 & 25 & 12,8 & 0,017 \\
\hline & $\begin{array}{c}\text { Una vez al } \\
\text { día }\end{array}$ & 6 & 15,4 & 5 & 38,5 & 8 & 47,1 & 26 & 20,5 & 45 & 23,0 & 0,169 \\
\hline & $\begin{array}{c}2 \text { a } 3 \\
\text { veces al } \\
\text { día }\end{array}$ & 6 & 15,4 & 1 & 7,7 & 1 & 5,9 & 15 & 11,8 & 23 & 11,7 & 0,796 \\
\hline & $\begin{array}{l}\text { Más de } 3 \\
\text { veces al } \\
\text { día } \\
\end{array}$ & 0 & 0,0 & 0 & 0,0 & 1 & 5,9 & 3 & 2,4 & 4 & 2,0 & 0,515 \\
\hline \multirow{6}{*}{$\begin{array}{l}\text { Consistencia } \\
\text { de las heces }\end{array}$} & Muy duro & 13 & 33,3 & 0 & 0,0 & 0 & 0,0 & 0 & 0,0 & 0 & 0,0 & \multirow[t]{6}{*}{0,000} \\
\hline & Duro & 26 & 66,7 & 0 & 0,0 & 0 & 0,0 & 0 & 0,0 & 26 & 13,3 & \\
\hline & $\begin{array}{c}\text { No tan } \\
\text { duro, ni } \\
\text { tan blando }\end{array}$ & 0 & 0,0 & 0 & 0,0 & 0 & 0,0 & 127 & 100,0 & 127 & 64,8 & \\
\hline & $\begin{array}{c}\text { Blando o } \\
\text { muy } \\
\text { blando }\end{array}$ & 0 & 0,0 & 9 & 69,2 & 0 & 0,0 & 0 & 0,0 & 9 & 4,6 & \\
\hline & Líquido & 0 & 0,0 & 4 & 30,8 & 0 & 0,0 & 0 & 0,0 & 4 & 2,0 & \\
\hline & $\begin{array}{c}\text { Variable } \\
\text { (Las heces } \\
\text { no } \\
\text { siempre } \\
\text { son } \\
\text { iguales) }\end{array}$ & 0 & 0,0 & 0 & 0,0 & 17 & 100,0 & 0 & 0,0 & 17 & 8,7 & \\
\hline \multicolumn{2}{|c|}{ Heces grandes } & 8 & 20,5 & 4 & 30,8 & 2 & 11,8 & 10 & 7,9 & 24 & 12,2 & 0,092 \\
\hline \multicolumn{2}{|c|}{ Postura de retención } & 13 & 33,3 & 7 & 53,9 & 10 & 58,8 & 37 & 29,1 & 67 & 34,2 & 0,308 \\
\hline \multicolumn{2}{|c|}{ Dolor al defecar } & 24 & 61,5 & 10 & 76,2 & 9 & 52,9 & 44 & 34,7 & 87 & 44,4 & 0,136 \\
\hline \multicolumn{2}{|c|}{ Fuerza para defecar } & 26 & 66,7 & 9 & 69,2 & 6 & 35,3 & 44 & 34,7 & 85 & 43,4 & 0,114 \\
\hline \multicolumn{2}{|c|}{ Urgencia para defecar } & 19 & 48,7 & 11 & 84,6 & 9 & 52,9 & 59 & 46,5 & 98 & 50,0 & 0,586 \\
\hline \multicolumn{2}{|c|}{ Tenesmo } & 21 & 53,9 & 10 & 76,9 & 12 & 71,0 & 40 & 31,5 & 83 & 42,4 & 0,059 \\
\hline \multicolumn{2}{|c|}{ Incontinencia fecal } & 1 & 2,6 & 4 & 30,8 & 2 & 11,8 & 7 & 5,5 & 14 & 7,1 & 0,020 \\
\hline \multicolumn{2}{|c|}{ Heces mucosas } & 8 & 20,5 & 4 & 30,8 & 5 & 29,4 & 14 & 11,0 & 31 & 15,8 & 0,168 \\
\hline \multicolumn{2}{|c|}{ Fecalomas } & 4 & 10,3 & 6 & 46,2 & 0 & 0,0 & 5 & 3,9 & 15 & 7,7 & 0,000 \\
\hline
\end{tabular}

*Chi cuadrado. 
Tabla 4. Características digestivas y extradigestivas de acuerdo a los subtipos de síndrome de intestino irritable en niños ( $\mathrm{N}=196)$.

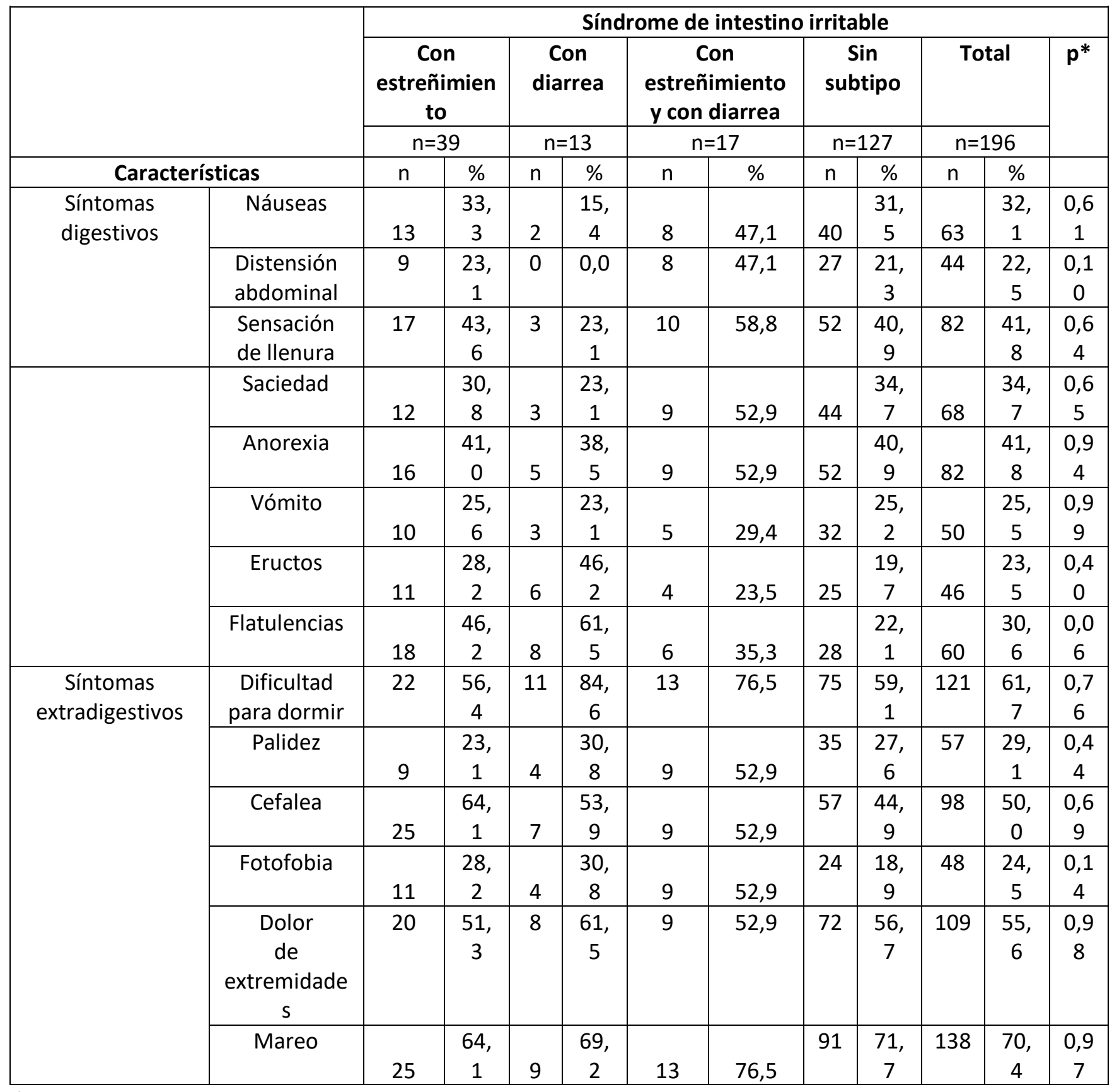

*Chi cuadrado.

No se encontraron posibles asociaciones entre los niños con SIl y los grupos de edad ( $R P=0,74$ IC $95 \%=0,36-1,47 \quad p=0,36)$, el sexo $(R P=1,09 \quad$ IC $95 \%=0,58-2,04 \quad \mathrm{p}=0,77)$, la ciudad ( $R P=0,50$ IC $95 \%=0,26-1,44 \quad p=0,20)$, la región ( $R P=1,01$ IC $95 \%=0,53-1,93 \quad p=0,97)$, los DGF intrafamiliares $(R P=1,36$ IC95\%=0,45-3,96 $p=0,52)$, ser hijo único
( $\mathrm{RP}=0,74$ IC $95 \%=0,19-2,39 \mathrm{p}=0,58)$, tener padres separados/divorciados ( $\mathrm{RP}=0,96$ IC $95 \%=0,50-1,82$ $\mathrm{p}=0,89)$, ser malnutrido ( $R P=1,31 \mathrm{IC} 95 \%=0,66-2,56$ $\mathrm{p}=0,38)$ o tener talla alterada para la edad $(\mathrm{RP}=0,43$ IC $95 \%=0,07-1,68 \mathrm{p}=0,19)$. Por otra parte, al analizar los subtipos de SII se hallaron posibles asociaciones entre los niños con Sll-d y ciudad 
( $R P=0,18$ IC $95 \%=0,04-0,73 \mathrm{p}=0,00)$ y malnutrición ( $R P=3,60$ IC $95 \%=0,98-14,56 p=0,02)$.

\section{DISCUSIÓN}

En Latinoamérica no han sido descritos para pediatría estudios sobre hábitos intestinales y los síntomas de acuerdo a los diferentes subtipos del SII. En esta investigación, más de la mitad de los niños presentaron SII-ss; una cuarta parte, SII-e, y el SII-m y el SII-d tuvieron una distribución similar. Rajindrajith y Devanarayana ${ }^{15}$, en 107 niños de Sri Lanka (edad promedio 12,9 $\pm 1,8$ años, 59,8\% femeninos) que reunieron los Criterios de Roma III para SII, describen de manera equitativa el SII-e, el SII-d y el SII-m (27,0\%-28,0\%), mientras que el SII-ss el menos prevalente (17,8\%). Devanarayana et al. ${ }^{16}$, en el mismo país, y para 427 niños, $50,1 \%$ del género masculino, con edad promedio $14,4 \pm 1,3$ años, reportan según los Criterios de Roma II predominio del SII-e en una tercera parte, seguidos en una cuarta parte por el SII-d y el SII-ss. Giannetti et $a l .{ }^{17}$, en un seguimiento a 100 niños italianos por un año (edad promedio 9,9 años, rango 4,2-16,7 años, 52\% niñas), reportan según los Criterios de Roma III el subtipo SII-e en un $45,0 \%$, el SII-m en un $29,0 \%$ y el SII-d en un $26,0 \%$. Self et al. ${ }^{18}$, en 129 niños norteamericanos con SIl según los Criterios de Roma III, entre 7 y 18 años de edad (promedio $11,4 \pm 2,8$ años, $60,5 \%$ niñas, $69,0 \%$ caucásicos), reportan SII-e en el 58,1\%, SII-ss en el 34,1\%, SII-d en el $5,4 \%$ y SII-m en el 2,3\%. Estas diferencias de resultados se deben probablemente a que el curso clínico del SII es bastante heterogéneo, ya que difiere dependiendo de la población evaluada, de la localización geográfica y de los criterios usados para definir SII y los subtipos de hábitos intestinales.

El SIl es una de las principales causas de dolor abdominal en niños con DGF. En este estudio, de los 4.394 cuestionarios analizados de la base de datos del Grupo FINDERS por Criterios de Roma III, 196 niños presentaron SII (4,5\%), siendo la segunda causa de DGF, seguido de la principal causa de DGF: el estreñimiento funcional $(11,6 \%)^{2}$. Estos datos de prevalencia del SII son similares a los descritos en países latinoamericanos como México $(6,4 \%)^{19}$, El Salvador $(3,8 \%)^{20}$, Nicaragua $(1,0 \%)^{21}$, Panamá $(5,6 \%)^{22}$, Ecuador $(4,8 \%)^{23}$ y Colombia $(5,4 \%)^{24}$.
En esta investigación, el SII fue más prevalente en las niñas que en los niños, pero sin diferencias significativas $(p=0,77)$, igual a lo descrito por Self et al. ${ }^{18}$, pero diferente a lo reportado por Rajindrajith y Devanarayana ${ }^{15}$, quienes encuentran diferencias significativas en el género $(59,8 \%$ niñas vs. $40,2 \%$ niños, $p=0,00)$, y por Giannetti et al. ${ }^{17}$, quienes observan predominio del género femenino en los niños con SIl-e y del género masculino en los niños con SII-d. Al igual que Rajindrajith y Devanarayana ${ }^{15}$, en el presente estudio se halló una reducción lineal en la probabilidad de desarrollar SII a medida que los individuos crecen, tanto en niñas como en niños. Con relación al sexo y a la edad, Lu et al. ${ }^{6}$, al estudiar 187 niños colombianos entre los 8 y 18 años con SIl según los Criterios de Roma III (11,6 62,4 años, 51,9\% niñas), no señalaron predominio entre el género, pero sí en cuanto al grupo de edad, cuya prevalencia fue mayor en los escolares (8-12 años) que en los adolescentes (13-18 años) con SII.

En la presente investigación no se encontraron diferencias significativas con respecto a la severidad del dolor abdominal, al igual que lo descrito por Self et al. ${ }^{18}$, pero diferente a lo comunicado por Rajindrajith y Devanarayana ${ }^{15}$, quienes registraron mayor dolor abdominal en los niños con SII-d $(p=0,02)$.

Así mismo, mientras que Rajindrajith y Devanarayana $^{15}$ encuentran que síntomas como fuerza y urgencia para defecar, tenesmo y heces mucosas son comunes en los niños con todos los subtipos de SII, en esta investigación la incontinencia fecal $(p=0,02)$ y los fecalomas $(p=0,00)$ fueron estadísticamente significativos.

En cuanto a los síntomas digestivos y extradigestivos reportados en los niños de este trabajo, no se encontraron diferencias significativas en ellos $(p>0,05)$, igual que lo reportado por Giannetti et $a{ }^{17}{ }^{17}$, quienes describen en un $41,0 \%$ dificultad para dormir, en $32,0 \%$ dolor de extremidades y en $43,0 \%$ cefalea. A su vez, estos hallazgos varían con respecto a lo descrito por Rajindrajith et $a l .{ }^{15}$, quienes señalan que los niños con SII presentaron más distensión abdominal, flatulencias, eructos, cefalea y dolor de extremidades $(p<0,05)$. Saps et $a .^{25}$, por su parte, en 53 niños con SII según los Criterios de Roma III, 
de tres países latinoamericanos y entre 8 y 15 años de edad $(11,7 \pm 2,1$ años, $58,5 \%$ femenino), reportan náuseas en el 32,0\%: El Salvador (46,7\%), Panamá $(33,3 \%)$ y Ecuador $(50,0 \%)$.

En los escolares y adolescentes de este trabajo, cerca del $46,0 \%$ faltaron al colegio o dejaron de hacer sus actividades diarias, sin diferencias significativas, igual a lo descrito por Giannetti et al. ${ }^{17}$, quienes reportan $53,0 \%$ de ausencias escolares y/o interrupción de actividades diarias $(p>0,05)$, pero diferente a lo relatado por Rajindrajith y Devanarayana ${ }^{15}$, quienes afirman que un $46,7 \%$ de los niños con SII faltaron al colegio al menos una vez durante los últimos dos meses $(p<0,0001)$.

Esta investigación es de corte transversal y no identificó cambios en los subtipos del SII durante el tiempo. Giannetti et al. ${ }^{17}$ por otra parte reportan, en el $24,2 \%$ de pacientes que siguieron durante un año, cambios en los subtipos, siendo principalmente observado el de SII-m hacia SII-e o SII-d.

En este trabajo se encontraron 3,6 veces más oportunidades de que se presente malnutrición en los niños con SII-d, lo que deberá a futuro obligar a realizar un seguimiento estrecho de estos individuos ante la posibilidad de que sea el inicio de alguna enfermedad de tipo orgánico. Así mismo se establece además la necesidad de realizar estudios de causalidad que busquen la asociación entre SII y malnutrición. En niños colombianos con estreñimiento funcional, no se encontró asociación entre sobrepeso, obesidad y el desorden gastrointestinal funcional ${ }^{26}$.

La fortaleza de este estudio radica en la inclusión de niños de colegios públicos y privados de diversos municipios y ciudades del país, así como la metodología sistemática llevada a cabo en investigaciones previas por el Grupo FINDERS (Functional International Digestive Epidemiological Research Survey), que permite comparaciones entre estudios. Por otro lado, entre las limitaciones de la investigación está la no realización de la Escala de Bristol (BSS) para identificar la forma de las heces y el no seguimiento por un tiempo de los niños, datos que ayudan a una mejor clasificación de los subtipos del SII. Sin embargo, con relación a la BSS para identificar la forma de las heces,
Vriesman et al. ${ }^{27}$, al estudiar a 1.835 niños colombianos entre los 8 y 18 años de edad $(12,8 \pm 2,3$ años, $51,6 \%$ masculinos), encuentran una concordancia moderada $(k=0,046 ; p=0,02)$ entre el QPGS-III y la BSS en la evaluación de la consistencia de las heces en niños, de manera que se sugiere la necesidad de mejores instrumentos para evaluar la consistencia de las heces con un alto grado de confiabilidad, tanto en investigación como en el entorno clínico. Otra de las debilidades del estudio se encuentra en el uso de chi cuadrado para el análisis estadístico de la asociación de las variables categóricas, pues la estimación se limita cuando los valores son inferiores a cinco, tal como los analizados en las tablas presentadas. Por lo tanto, hay que interpretar los valores de $\mathrm{p}$ con cuidado, pues están sesgados debido a dicha restricción.

En conclusión, luego del SII-ss, una cuarta parte de los escolares y adolescentes estudiados presentó SII-e, seguido del SII-m y del SII-d. Este último fue el que se presentó con mayor oportunidad en los niños malnutridos.

\section{DECLARACIÓN SOBRE CONFLICTO DE INTERESES}

Los autores manifiestan que son independientes con respecto a las instituciones de apoyo y que durante la ejecución del trabajo o la redacción del manuscrito no han incidido intereses o valores distintos a los que usualmente tiene la investigación. Los autores declaran no tener ningún conflicto de interés.

\section{CONTRIBUCIÓN DE LOS AUTORES}

Todos los autores participaron por partes iguales en cuanto al trabajo de campo, la redacción, el diseño metodológico, el análisis estadístico, la construcción de la base de datos, la redacción y la elaboración del manuscrito.

\section{REFERENCIAS BIBLIOGRÁFICAS}

1. Hyams JS, Di Lorenzo C, Saps M, Shulman RJ, Staiano A, van Tilburg M. Childhood Functional Gastrointestinal Disorders: Child/Adolescent. Gastroenterology. 2016; 150(6): 1456-68. Doi: https://doi.org/10.1053/j.gastro.2016.02.015. 
2. Saps M, Moreno-Gómez JE, Ramírez-Hernández CR, Rosen JM, Velasco-Benítez CA. A nationwide study on the prevalence of functional gastrointestinal disorders in school-children. Bol Med Hosp Infant Mex. 2017; 74(6): 407-412. Doi: https://doi.org/10.1016/j.bmhimx.2017.05.005.

3. Devanarayana NM, Mettananda S, Liyanarachchi C, Nanayakkara N, Mendis N, Perera N, et al. Abdominal Pain-Predominant Functional Gastrointestinal Diseases in Children and Adolescents: Prevalence, Symptomatology, and Association With Emotional Stress. J Pediatr Gastroenterol Nutr. 2011; 53(6): 659-65. Doi: https://doi.org/10.1097/MPG.0b013e318229603 3.

4. Saps M, Adams P, Bonilla S, Chogle A, NicholsVinueza D. Parental Report of Abdominal Pain and Abdominal Pain-related Functional Gastrointestinal Disorders From a Community Survey. J Pediatr Gastroenterol Nutr. 2012; 55(6): 707-10.

Doi: https://doi.org/10.1097/MPG.0b013e318266240 1.

5. Velasco-Benítez $C A$, Saps $M$, Chanís $R$, Játiva $E$, Zablah $\mathrm{R}$, Mejía $\mathrm{M}$, et al. La epidemiología de los desórdenes gastrointestinales funcionales en escolares y adolescentes latinoamericanos. Grupo de trabajo de la Sociedad Latinoamericana de Gastroenterología, Hepatología y Nutrición Pediátrica (SLAGHNP). Acta Gastroenterol Latinoam [revista en la internet]. 2017 [citado 24 jun 2019]; 47(2): 148-58. Disponible en: http://actagastro.org/la-epidemiologia-de-losdesordenes-gastrointestinales-funcionales-enescolares-y-adolescentes-latinoamericanosgrupo-de-trabajo-de-la-sociedadlatinoamericana-de-gastroenterologiahepatologia-y-nutr/.

6. Lu PL, Velasco-Benítez CA, Saps M. Sex, Age, and Prevalence of Pediatric Irritable Bowel Syndrome and Constipation in Colombia: A Populationbased Study. J Pediatr Gastroenterol Nutr. 2017; 64(6): e137-41. Doi: https://doi.org/10.1097/MPG.000000000000139 1.
7. Ersryd A, Posserud I, Abrahamsson H, Simren M. Subtyping the irritable bowel syndrome by predominant bowel habit: Rome II versus Rome III. Aliment Pharmacol Ther. 2007; 26(6): 953-61. Doi: https://doi.org/10.1111/j.13652036.2007.03422.x.

8. Yao X, Yang YS, Cui LH, Zhao KB, Zhang AH, Peng $\mathrm{LH}$, et al. Subtypes of irritable bowel sindrome on Rome III criteria: A multicenter study. J Gastroenterol Hepatol. 2012; 27(4): 760-5. Doi: https://doi.org/10.1111/j.1440-

1746.2011.06930.x.

9. Heitkemper M, Cain KC, Shulman R, Burr R, Poppe A, Jarrett $M$. Subtypes of Irritable Bowel Syndrome Based on Abdominal Pain/Discomfort Severity and Bowel Pattern. Dig Dis Sci. 2011; 56(7): 2050-8. Doi: https://doi.org/10.1007/s10620-011-1567-4.

10. Walter SA, Skagerstrom E, Bodemar G. Subgroups of irritable bowel syndrome: a new approach. Eur J Gastroenterol Hepatol [revista en la internet]. 2004 [citado 24 jun 2019]; 16(10): 991-4. Disponible en: https://journals.lww.com/eurojgh/pages/articlevi ewer.aspx ?year $=2004 \&$ issue $=10000 \&$ article $=000$ $07 \&$ type=abstract.

11. Kibune-Nagasako C, García-Montes C, SilvaLorena SL, Aparecida-Mesquita M. Irritable bowel syndrome subtypes: Clinical and psychological features, body mass index and comorbidities. Rev Esp Enferm Dig [revista en la internet]. 2016 [citado 24 jun 2019]; 108(2): 59-64. Disponible en:

https://online.reed.es/Revistas/REED_2016_108_ 2/Contenido/pdf/vol108num2_en_2.pdf.

12. Keshtelia $A H$, Dehestania $B$, Daghaghzadeha $H$, Adibia P. Epidemiological features of irritable bowel syndrome and its subtypes among Iranian adults. Ann Gastroenterol [revista en la internet]. 2015 [citado 24 jun 2019]; 28(2): 253-8. Disponible en: https://www.ncbi.nlm.nih.gov/pmc/articles/PMC 4367216/.

13. Rasquin A, Di Lorenzo C, Forbes D, Guiraldes E, Hyams JS, Staiano A, et al. Childhood Functional Gastrointestinal Disorders: Child/Adolescent. 
Gastroenterology. 2006; 130: 1527-37. Doi: https://doi.org/10.1053/j.gastro.2005.08.063.

14. Saps M, Nichols-Vinueza DX, Mintjens S, Pusatcioglu CK, Velasco-Benítez CA. Construct Validity of the Pediatric Rome III Criteria. J Pediatr Gastroenterol Nutr. 2014; 59: 577-81. Doi: https://doi.org/10.1097/MPG.000000000000048 2.

15. Rajindrajith S, Devanarayana NM. Subtypes and Symptomatology of Irritable Bowel Syndrome in Children and Adolescents: A School-based Survey Using Rome III Criteria. J Neurogastroenterol Motil. 2012; 18(3): 298-304. Doi: https://doi.org/10.5056/jnm.2012.18.3.298

16. Devanarayana NM, Adhikari C, Pannala W, Rajindrajith S. Prevalence of Functional Gastrointestinal Diseases in a Cohort of Sri Lankan Adolescents: Comparison Between Rome II and Rome III Criteria. J Trop Pediatr. 2011; 57(1): 34-9. Doi: https://doi.org/doi:10.1093/tropej/fmq039

17. Giannetti E, de'Angelis G, Turco R, Campanozzi A, Pensabene L, Salvatore $S$, et al. Subtypes of Irritable Bowel Syndrome in Children: Prevalence at Diagnosis and at Follow-Up. J Pediatr. 2014; 164(5): 1099-1103. Doi: https://doi.org/10.1016/j.jpeds.2013.12.043.

18. Self MM, Czyzewski DI, Chumpitazi BP, Weidler EM, Shulman RJ. Subtypes of Irritable Bowel Syndrome in Children and Adolescents. Clin Gastroenterol Hepatol. 2014; 12(9): 1468-73. Doi: https://doi.org/doi:10.1016/j.cgh.2014.01.031.

19. Dhroove G, Saps M, García-Bueno C, Leyva Jiménez A, Rodríguez-Reynosa LL, Velasco-Benítez CA. Prevalencia de trastornos gastrointestinales funcionales en escolares mexicanos. Rev Gastroenterol Mex. 2017; 82(1): 13-8. Doi: https://doi.org/10.1016/j.rgmx.2016.05.003.

20. Zablah R, Velasco-Benítez CA, Merlos I, Bonilla S, Saps M. Prevalencia de trastornos funcionales gastrointestinales en niños en edad escolar en $\mathrm{El}$ Salvador. Rev Gastroenterol Mex. 2015; 80(3): 186-91.

https://doi.org/10.1016/j.rgmx.2015.03.008.
21. Mejía M, Velasco-Benítez CA, Díaz J. La prevalencia y las posibles asociaciones de los desórdenes gastrointestinales funcionales en escolares y adolescentes de colegios privados de Managua, Nicaragua. Acta Gastroenterol Latinoam [revista en la internet]. 2017 [citado 24 jun 2019]; 47(3): 163-8. Disponible en: http://actagastro.org/la-prevalencia-y-lasposibles-asociaciones-de-los-desordenesgastrointestinales-funcionales-en-escolares-yadolescentes-de-colegios-privados-de-managuanicaragua/.

22. Lu PL, Saps M, Chanis RA, Velasco-Benítez CA. The prevalence of functional gastrointestinal disorders in children in Panama: a school-based study. Acta Pædiatrica. 2016; 105(5): e232-6. Doi: https://doi.org/10.1111/apa.13379.

23. Jativa E, Velasco-Benítez CA, Koppen IJN, JátivaCabezas Z, Saps M. Prevalence of Functional Gastrointestinal Disorders in Schoolchildren in Ecuador. J Pediatr Gastroenterol Nutr. 2016; 63(1): 25-8. Doi: https://doi.org/10.1097/MPG.000000000000110 8.

24. Saps M, Nichols-Vinueza DX, Rosen JM, VelascoBenítez CA. Prevalence of Functional Gastrointestinal Disorders in Colombian School Children. J Pediatr. 2014; 164(3): 542-5. Doi: https://doi.org/10.1016/j.jpeds.2013.10.088.

25. Saps M, Velasco-Benítez C, Kovacic K, Chelimsky $G$, Kovacic $C$, Játiva $E$, et al. High Prevalence of Nausea among School Children in Latin America. J Pediatr. 2016; 169(2): 98-104. Doi: https://doi.org/10.1016/j.jpeds.2015.10.052.

26. Koppen IJN, Velasco-Benítez CA, Benninga MA, Di Lorenzo C, Saps M. Is There an Association between Functional Constipation and Excessive Bodyweight in Children? J Pediatr. 2016; 171(4): 178-82.

Doi: https://doi.org/10.1016/j.jpeds.2015.12.033.

27. Vriesman $\mathrm{MH}$, Velasco-Benítez $\mathrm{CA}$, Ramírez $\mathrm{CR}$, Benninga MA, Di Lorenzo C, Saps M. Assessing Children's Report of Stool Consistency: Agreement Between the Pediatric Rome III Questionnaire and the Bristol Stool Scale. J Pediatr. 2017; 190(11): 69-73. Doi: https://doi.org/10.1016/j.jpeds.2017.07.002. 